\title{
Chemosensory conditioning in molluscs: II. A critical review
}

\author{
JOSEPH FARLEY, IKSUNG JIN, HAOJIANG HUANG, and JAE-IL KIM \\ Indiana University, Bloomington, Indiana
}

\begin{abstract}
We critically review chemosensory conditioning studies with molluscs and find that, in many studies, the influence of nonassociative processes complicates, obscures, and renders ambiguous the unique contribution of associative learning. These nonassociative processes include sensory adaptation, habituation, sensitization, and changes in feeding motivation. They arise from both the food extracts that have often been used as conditioned stimuli and the aversive stimuli that have been used as unconditioned stimuli.
\end{abstract}

As was described in the preceding article (Jin, Huang, Kim, \& Farley, 2004), we observed large increases in bite/strike latencies to both reinforced and nonreinforced chemosensory cues following contextual chemosensory conditioning of Hermissenda (cf. Rogers, Schiller, \& Matzel, 1996), thereby failing to obtain clear evidence for associative learning with this response measure.

We also failed to observe decreased preference to a food, in Y-maze tests of choice behavior, whose extract (reinforced conditioned stimulus; $\mathrm{CS}+$ ) had been contextually paired with rotation/shaking, as would be expected if the animals had acquired an aversion to the CS + . Only a small percentage of the animals exhibited meaningful and reliable choice behavior during the baseline test prior to conditioning. And even for these animals, no clear decrease in preference to the CS+ was observed during either of two postconditioning tests. The small decreases that did occur were not significantly different from those to a nonreinforced conditioned stimulus ( $\mathrm{CS}-$ ).

Both of these failures to obtain clear evidence for associative context conditioning to chemosensory cues for Hermissenda appear to reflect the contribution of several potent nonassociative behavioral processes. Here, we will elaborate on the nature, genesis, and interaction of these influences that arise in the chemosensory aversion paradigms used with Hermissenda and their implications for demonstrations of genuine associative learning. We next will discuss the contribution of similar processes to the chemosensory aversion paradigms used with other gastropod molluscs and will conclude that here, too, these influences can confound unambiguous demonstrations of associative learning.

I.J. and H.H. both contributed equally to this research and were affiliated with the Program in Neural Science at Indiana University, Bloomington. J.-I.K., who also was affiliated with the Program in Neural Science at Indiana University, was killed in an automobile accident on May 31, 1999. We dedicate this article and the one that precedes it to him. Correspondence concerning this article should be addressed to J. Farley, Graduate Programs in Neural Science and Biochemistry, Indiana University, Bloomington, IN 47405-7007 (e-mail: farleyj@indiana.edu).

\section{Nonassociative Sources of Feeding Suppression in Hermissenda}

Our experiments indicated that the use of concentrated food extracts as contextual cues produced large nonspecific increases in Hermissenda's bite/strike latencies to food CSs, as well as decreases in exploration and activity in a Y-maze (Jin et al., 2004). Although we have not yet characterized the different processes involved, we expect all of the following to contribute to differing degrees.

First, concentrated food extracts probably produce satiation-like decreases in feeding motivation similar to those produced when solid food is eaten. Animals actively fed on and swallowed extract-flavored artificial seawater (ASW) in our experiments. Indeed, many of the animals ingested so much extract-laced ASW that they were visibly bloated upon removal from the conditioning chambers. Food-satiated Hermissenda exhibit large elevations in feeding thresholds and frequently show an active aversion to food (Ram, Noirot, Waddell, \& Anderson, 1988). In other molluscs (e.g., Lymnaea), satiation has also been shown to disrupt both the acquisition (Audesirk, Alexander, Audesirk, \& Moyer, 1982; Kemenes \& Benjamin, 1994) and the expression (Audesirk et al., 1982) of conditioned feeding responses. It seems likely that similar interference effects occurred here with Hermissenda. Because the presence of food suppresses a variety of active movements in Hermissenda, including phototactic behavior in a light gradient (Alkon, Akaike, \& Harrigan, 1978) and spontaneous locomotion and roll-over behavior (Ram et al., 1988), it is likely that extract exposure contributed to the decreased Y-maze behavior that we observed following contextual conditioning. We do not yet know the cues that regulate feeding in Hermissenda, but it seems safe to assume that "hunger" in Hermissenda is a multiple-input/multiple-output system (Mook, 1990). Thus, we suspect that the animals' ingestion of the extracts stimulated both internal (e.g., esophageal distension and nutrient levels) and external (e.g., taste) processes that inhibit feeding, as has commonly been observed for many species of animals. In short, we think that satiety produced behavioral tranquilization. 
A second set of processes likely to have been engaged by prolonged food extract exposures is adaptation at early stages in chemosensory processing, especially decreased chemosensitivity occurring peripherally (i.e., outside the central nervous system [CNS]). Chemosensory adaptation in gastropods encompasses not only decreases in chemoreceptor sensitivity during the presence of a maintained stimulus (i.e., altered sensory transduction), but also pre- and postsynaptic changes in gustatory coding and integration in peripheral second-order neurons. A general feature of both odorant- and tastestimulated signals mediated by G-protein coupled receptors, common to both vertebrates and invertebrates (Hildebrand \& Shepherd, 1997), is rapid desensitization in the continual presence of a chemosensory stimulus. Cross-desensitization effects are common in chemosensory systems, particularly when prolonged and/or concentrated stimuli are used, and are a potential source of generalized response decrements of the sort observed here.

A third probable influence involves the combined effects of habituation and sensitization on feeding reflexes: changes within and between the interneurons, premotor neurons, and motoneurons that mediate ingestion behaviors.

Finally, a complete list of nonassociative factors likely to have influenced our tests of Hermissenda's appetitive behaviors would include several unrelated to chemosensory stimulation, such as the effects of mechanosensory and vestibular stimulation. Both animal handling (Grover, Farley, \& Vold, 1987) and orbital rotation/shaking (Alkon, 1974) suppress locomotion and, thus, are likely to have contributed to the inactivity of the animals in the posttraining Y-maze tests. They may also have contributed to suppressed feeding reflexes.

In summary, the bite/strike response latency increases that we observed following contextual chemosensory conditioning of Hermissenda (Jin et al., 2004) probably arose from a panoply of nonassociative effects, including satiation, sensory adaptation, and habituation processes. These are attributable to the prolonged exposure to the chemosensory stimulus that is characteristic of contextual conditioning. During postconditioning Y-maze tests, these nonassociative processes probably produced animals that were generally inactive, failed to sample both alternatives in the maze, and also failed to contact food at the end of a selected baited arm. These potent nonassociative effects can be expected to (1) obscure and/or prevent the occurrence of associative learning and (2) complicate interpretation of changes in chemosensory-evoked behaviors as reflecting associative-learning processes.

\section{Additional Reports of Contextual Conditioning in Hermissenda}

Two other reports of context conditioning with Hermissenda have appeared. Rogers and Matzel (1995) used chemosensory cues as contextual stimuli and training methods essentially identical to those in Rogers et al.
(1996). No direct measures of conditioning to the chemosensory cue that signaled shaking were reported in this study (e.g., changes in feeding latencies, preference for the reinforced chemosensory contextual cue in choice tests, etc.). Instead, the occurrence of contextual conditioning was inferred from indirect evidence: the ability of the reinforced chemosensory cue to "block" subsequent excitatory conditioning to light, when light was paired with orbital rotation in the presence of the chemosensory cue. Conditioning to light was assessed by the extent of pedal musculature contraction (i.e., clinging) that light elicited, when later tested alone.

We have not attempted to repeat these contextual blocking results and, thus, cannot comment on their reliability. However, in discrete-trial chemosensory blocking and overshadowing experiments, we have observed opposite effects: Chemosensory stimuli potentiate conditioning to light (Farley \& Jin, 1997; Farley, Reasoner, \& Janssen, 1997). Nonetheless, accepting Rogers and Matzel's (1995) results at face value, we have previously discussed in detail their possible interpretation and significance (Farley et al., 1997, pp. 336-338). It will suffice to note here that in addition to the associative explanation of blocking advanced by Rogers and Matzel, several nonassociative accounts are also consistent with their results. The one that is most germane to the issues considered here questions whether an association was formed between the contextual chemosensory cue and shaking during the initial phase of training in the blocking experiments. As an alternative, we proposed an account that invoked the nonassociative processes of habituation, sensitization, and protection from habituation, coupled with the idea that an association is formed between light and the chemosensory stimulus that then opposes that association formed between light and shaking.

For example, perhaps prolonged (25-min) exposures to scallop extract (in the absence of shaking) result in chemosensory adaptation, habituation, satiation, and so forth, which combine to diminish CNS responses to scallop. However, if pulses of shaking are repeatedly administered, these nonassociative processes are attenuated. Animals may even be sensitized by the vigorous shaking. It is worth emphasizing that the chemosensory cue (scallop) is not a neutral CS but is a biologically important unconditioned stimulus (US) and may well be able to support conditioning in its own right. Therefore, we suggest that during the second phase of the blocking experiments, when animals were exposed to compound pairings of light, scallop extract (previously used to signal shaking), and orbital rotation, they not only were forming an association between light and rotation, but were also associating light with scallop. The association between light and shaking supported a foot contraction conditioned response (CR). But because scallop extract, like other foods, is an appetitive stimulus, eliciting approach behavior and foot extension, we think it likely that the association between light and scallop supported an opposing foot extension (CR) that antagonized foot 
contraction. In this account, the apparent "blocking" of conditioning to light does not reflect a failure of learning about light. On the contrary, the animal has learned two, mutually antagonistic responses. In contrast, control animals that received nonreinforced scallop exposures during the first phase of training, followed by compound light, scallop, and rotation pairings [designated as groups $(\mathrm{A}-) \mathrm{A}: \mathrm{P}$ and $(\mathrm{IO}+/ \mathrm{A}-) \mathrm{A}: \mathrm{P}$ from Experiments 1 and 2 of Rogers \& Matzel (1995)] showed foot contraction to light due to the unopposed light-shaking association.

More recently, Talk, Muzzio, and Matzel (1999) reported contextual conditioning to an environment whose distinguishing feature was that it was continuously illuminated. Hermissenda confined to one half of an illuminated alleyway for $15 \mathrm{~min}$ (the lit context) and exposed therein to repeated, brief, unsignaled, orbital rotations later avoided this lit context in a choice test and increased their time spent in the other half of the alleyway, which was dark. Talk et al. concluded that their animals had associated light with shaking, and since light had been continuously present, it could be considered a cue analogous to the static, unchanging stimuli that are conventionally taken to define the environment in more traditional contextconditioning studies. The report of an in vitro neural analogue of lit context conditioning in the Type B photoreceptors, similar to that produced by discrete-trial lightrotation pairings, considerably strengthens Talk et al.'s claim to have demonstrated contextual conditioning to continuous illumination.

However, some caution is probably warranted before considering this demonstration conclusive. First, the nature of the choice test used makes it ambiguous as to whether the animals' altered light-dark preferences resulted from increased avoidance of light, increased attraction to the dark, or both. Thus, it is unclear as to how the learning that occurred should be characterizednamely, excitatory conditioning to light or inhibitory conditioning to dark. Although it might be argued that either way, the results demonstrate context conditioning, only the former type of learning would be consistent with Talk et al.'s (1999) hypothesized neurophysiological account of lit context conditioning.

Second, the distinction between a discrete and a contextconditioning trial may break down in the training paradigm used by Talk et al. (1999), where 60 repeated pulses of $3 \mathrm{sec}$ of orbital rotation were delivered every $15 \mathrm{sec}$ (a relatively high frequency). Vigorous orbital rotation dislodges animals from their substrate (Alkon, 1974), subjecting them to considerable turbulence and mechanosensory stimulation that outlasts the termination of rotation. From Figure 2 in Rogers et al. (1996), animals shaken for $3 \mathrm{sec}$ at $300 \mathrm{rpm}$ - the same stimulation parameters as those used in Talk et al.- - lost their grip on the substrate after $\sim 1 \mathrm{sec}$ and were tossed, tumbled, and banged about their conditioning chambers for some time afterward (at least $10 \mathrm{sec}$ or more, when we checked in our own conditioning apparatus). Therefore, although each orbital rotation US lasted only a nominal $3 \mathrm{sec}$, the actual duration of vestibular and mechanosensory stimulation was considerably longer. Once the animals were dislodged, it is unclear whether they reattached to the substrate in Talk et al.'s experiments. This suggests that the animals' experience in these experiments was one where continuous illumination occurred conjointly with prolonged and relatively uninterrupted turbulence, punctuated by periodic, brief, high-speed orbital rotation. From this perspective, the conditioning paradigm may be construed as consisting of several extended "simultaneous" conditioning trials in which illumination and turbulence overlap (albeit their onsets were delayed by 7-8 sec). Although Talk et al. justified their use of short US interstimulus intervals (ISIs) on the basis of findings with vertebrates that contextual conditioning is facilitated for massed versus distributed USs, the USs used in those studies (electric shock in Williams, Frame, \& LoLordo, 1991; small rat chow pellets that were immediately consumed or 4-sec pigeon grain presentations in Mustaca, Gabelli, Papini, \& Balsam, 1991) were brief and probably lasted little longer than their nominal presentation durations.

In summary, the available evidence concerning chemosensory contextual conditioning in Hermissenda is scant and conflicting, and the stimulus specificity of the effects reported for the most direct response measure (bite/ strike latency) is quite small. Methodological difficulties, unproven assumptions, and neglected alternative interpretations should encourage considerable skepticism as to whether its occurrence has yet been conclusively demonstrated.

\section{Nonassociative Influences in Chemosensory Learning Paradigms With Other Molluses}

Chemosensory learning paradigms with gastropod molluscs have generally used USs that can be categorized as aversive (e.g., strong electric shock or unpleasant chemical stimuli), appetitive (palatable food), or more complex social stimuli (e.g., pheromonal stimulation). There are ample grounds for believing that various nonassociative effects evoked by these USs can change feeding behavior in ways that are difficult to distinguish from those of associative learning. A salient feature of most of the aversive/avoidance learning paradigms used with molluscs is that they often produce a rather nonselective suppression of feeding.

Consider, for example, the experiments by Davis et al. (1980) on the carnivorous gastropod Pleurobranchaea. Historically, these and related studies provided some of the earliest and most impressive evidence for robust and persistent learning-induced changes in the feeding behaviors of molluscs and were influential in spurring the development of molluscan preparations as model systems for the study of associative learning and memory. In the first (single-stimulus training) experiment in Davis et al. (1980), food (e.g., crude squid homogenate) was applied to the animal's chemosensitive oral veil. If a feeding response occurred within $90 \mathrm{sec}$ (or if withdrawal failed to occur), a 60 -sec aversive electric shock 
was delivered. Ten to 20 of these pairing trials, spaced $1 \mathrm{~h}$ apart, were given over 1-2 days. Unpaired control animals were exposed to the same numbers and overall frequencies of extract presentations and electric shocks, but the two stimuli were separated by $30 \mathrm{~min}$. Several parameters of feeding and withdrawal behaviors were measured both before and after conditioning. These tests began with determination of response thresholds, defined as the minimum concentration of a food stimulus required to elicit the component of the feeding response of interest (e.g., proboscis extension or bite/strike behavior). Animals trained with squid as the $\mathrm{CS}+$ showed dramatically increased response latencies to squid, still apparent 8 days after training. The results of this study and earlier ones (Mpitsos, Collins, \& McClellan, 1978; Mpitsos \& Davis, 1973) were robust and clear. Prior to training, bite/strike latencies to squid were $\sim 2-3 \mathrm{sec} ; 1$ and 8 days following conditioning, the latencies were $\sim 75$ and $\sim 48 \mathrm{sec}$, respectively. For unpaired control animals, the latencies to squid at all posttraining retention intervals were essentially unchanged from the 2- to 3-sec baseline values. Thus, as assessed by a between-groups comparison, the changes to CS+ were exquisitely pairing specific.

However, when stimulus specificity was assessed by testing animals with untrained Corynactis (sea anemone) homogenate, substantially increased latencies were observed for both paired and unpaired groups. Latencies to Corynactis prior to conditioning were $\sim 8 \mathrm{sec}$ for both groups. One and 8 days after training, the latencies were $\sim 42$ and $\sim 15 \mathrm{sec}$ for paired animals and $\sim 30$ and $\sim 15 \mathrm{sec}$ for unpaired animals. Although the posttraining increases to squid were greater (for paired animals) than those to Corynactis, the latencies to Corynactis were clearly greater than baseline for both paired and unpaired animals. Thus, some factor common to both the paired- and the unpairedtraining conditions produced substantial increases in feeding response latencies, even to foods that had not been involved in training.

In their next experiment, which involved discrimination training, Davis et al. (1980) reported results for both a food homogenate $(\mathrm{CS}+)$ that had been paired with shock and a second, nonreinforced stimulus (CS-). This study is noteworthy in that it illustrates the extent to which the pairing and stimulus specificity of chemosensory learning in molluscs often depends on the particular foods chosen. Four replications were conducted, with the following assignment of food extracts to CS + and CS -, respectively: (1) squid and Corynactis, (2) Corynactis and squid, (3) shrimp and squid, and (4) squid and shrimp. Consistent and significant differences between the CS+ and the CS - were obtained when Corynactis (a nonpreferred food) was used as the $\mathrm{CS}+$ and squid (a highly preferred food) as the $\mathrm{CS}-$ (i.e., when the preconditioning food preferences were congruent with the expected outcome of associative learning). Despite this, the increases to the CS- (squid) were still $\sim 40 \%-60 \%$ of those occurring to the $\mathrm{CS}+$. The other stimulus combinations yielded a small nonsignificant trend toward differential increases to the $\mathrm{CS}+(\mathrm{CS}+=$ squid, $\mathrm{CS}-=$ shrimp $)$, nondifferential increases to both the CS+ (squid) and the CS- (Corynactis), or a trend where increases to the $\mathrm{CS}-$ (squid) were greater than those to the CS + (shrimp). As Davis et al. (1980) remarked, "significant differential learning occurred consistently only when CS + was Corynactis and the CS - was squid."

A comparison of the results obtained by Davis et al. (1980) for squid as the CS+ in the discrimination procedure (CS $-=$ Corynactis) versus the single-stimulus conditioning procedure is instructive and anticipates a pattern similar to the one we observed with Hermissenda in Experiments 1-3 of the accompanying article. Despite a gap of 30 min between Corynactis and shock during discrimination training, Pleurobranchaea showed as great an increase in bite/strike latencies to Corynactis as to squid. However, if trained only with squid as the CS+ (i.e., no CS - exposures given), the increases to Corynactis were only $\sim 40 \%-60 \%$ of those to squid. In short, stimulus specificity was minimized in the discriminationlearning paradigm (generalization was enhanced). This contrasts with the typical outcome of discriminative conditioning, for both vertebrates (Hanson, 1959; Jenkins \& Harrison, 1960, 1962; Moore, 1972; Rilling, 1977; Thomas, 1970) and various invertebrates (Bitterman, Menzel, Fietz, \& Schafer, 1983; Nelson, 1971; Sutherland \& Mackintosh, 1971) alike, where much more selective responding to a $\mathrm{CS}+$ than to a $\mathrm{CS}-$ is the usual result of discrimination training.

Davis et al. (1980) suggested that the strong aversion to the CS - for differentially conditioned animals was due to the animals' learning to avoid any food substance presented during aversive conditioning, including those not specifically coupled with shock. In their view, the presence of more than one food substance may have "confused" the animals as to which food should be avoided. The possibility that strong electric shock may disrupt the discriminative capacities of molluscs is very interesting but, unfortunately, has received little attention. It may be related to the phenomenon of generalized flavor neophobia following taste aversion conditioning in rats (Kristal, Steuer, Nishita, \& Peters, 1980).

At least three other interpretations of the pattern of poor discriminative control are possible. Variations of some of these were considered by Davis et al. (1980) but were found wanting. We will discuss each in turn. First, perhaps long-delay associative learning occurred to Corynactis when it was used as the CS- in differential conditioning. According to this interpretation, approximately equivalent associative learning occurred to squid and Corynactis, thus accounting for the lack of stimulus specificity. This hypothesis regards the strong aversions to a CS - in differentially conditioned specimens as reflecting a genuine association between the $\mathrm{CS}-$ and the US, rather than being an indirect consequence of either the "confusion" produced by strong electric shock or 
generalization of conditioning from the $\mathrm{CS}+$ to the $\mathrm{CS}-$ (due to common stimulus elements, of which there were plenty). The long-delay learning hypothesis is weakened by the absence of detectable conditioning to a shrimp CS - in unpaired control animals, when presented with shock at a 30-min ISI (Figures 2C and 2D in Davis et al., 1980). However, the result might have been different had Corynactis (a relatively nonpreferred food) been used.

A second interpretation of the lack of stimulus specificity for differentially conditioned animals is that shock suppressed feeding to all food substances because of changes in feeding motivation. Suppression of feeding by strong electric shock has been observed for several molluscs, including Aplysia (Kupfermann \& Pinsker, 1968) and Hermissenda (Farley, 1987). And aversive chemical stimulation $\left(\mathrm{CO}_{2}\right.$ toxicosis $)$ produces a general suppression of feeding in Limax (Gelperin, 1975). Can changes in feeding motivation explain the results for Pleurobranchaea? Davis et al. (1980) thought not, since earlier studies (Davis, Mpitsos, \& Pinneo, 1974) in which this issue had been examined directly failed to observe feeding suppression by electric shock. Some caution is called for in accepting this conclusion, however, since the shock parameters examined in the 1974 study were less severe than those used in 1980.

Although we cannot rule out entirely the above possibilities as contributing to the results from Davis et al. (1980), as well as our own with Hermissenda (Jin et al., 2004, Experiments 1-3), they would not explain our results in Experiments 4 and 5 (Jin et al., 2004). This is because the preceding hypotheses view the occurrence of the strongly aversive US, in one way or another, as critical for the increased latencies. Yet we obtained comparable response latency increases to two foods after repeated extended exposures to just one of them, even when no US presentations were given (Experiment 4). Furthermore, extended exposures to an orbital rotation US failed to increase response latencies to nonexposed shrimp and scallop when these were tested $24 \mathrm{~h}$ later (Experiment 5).

These considerations suggest a different interpretation of the lack of stimulus specificity. We propose that the additional chemosensory stimulation provided by $\mathrm{CS}-$ exposures contributed to the feeding declines. These decrements could have arisen from satiety, chemosensory adaptation, and/or habituation of feeding reflexes. In this view, the increases to the $\mathrm{CS}+$ could have primarily reflected associative learning due to pairing with the US, whereas the increases to the CS- arose mainly from nonassociative processes due to mere exposure to the chemosensory stimuli. Alternatively, the increases to both the $\mathrm{CS}+$ and the $\mathrm{CS}-$ could have reflected the nonassociative processes.

Although it might be argued that this view predicts that latencies to a CS + (squid) for differentially conditioned animals should also have been elevated, relative to those for animals trained only with a CS+ (Davis et al.,
1980), this prediction assumes that the contributions of nonassociative and associative learning to latency increases would summate. However, it seems just as likely that they might preclude one another. Habituation, sensory adaptation, and satiation might prevent associative learning from occurring (and perhaps vice versa). Consistent with this possibility is the abbreviated duration of the increases to the $\mathrm{CS}+$ for the differentially conditioned animals in Davis et al. (1980; CS $+=$ squid, CS $-=$ Corynactis). Latencies to the CS+ were elevated at the 1- and 2-day retention intervals but had declined back to baseline at 8 days (Figures 7A and 8A in Davis et al., 1980). In contrast, the latencies to the same CS + (squid) for the single-stimulus animals remained substantially elevated, even at the 8-day retention interval (Figures $2 \mathrm{~A}$ and $2 \mathrm{C}$ ). Evidently, the differential-conditioning procedure produced less enduring changes to the $\mathrm{CS}+$, in addition to less specific ones. This pattern is consistent with what one might expect if satiation/habituation/sensory adaptation were at work, as in our Experiment 4. Similarly, Mpitsos et al. (1978, Figure 13) reported that CS-alone presentations (squid extract) resulted in elevated thresholds for bite/strike behaviors in Pleurobranchaea, when assessed $12 \mathrm{~h}$ after training, but had returned to nearbaseline levels at a 24 -h retention interval.

Additional support for a major contribution of nonassociative processes to learned feeding suppression in Pleurobranchaea was provided by subsequent studies of neural correlates of chemosensory aversion learning. Gillette, Kovac, and Davis (1978) recorded intracellularly from the paracerebral command neurons ( $\mathrm{PCNs}$ ) key triggers of the feeding motor program (FMP) - of whole animal preparations previously exposed to either paired or unpaired squid-shock presentations, as well as from hungry or food-satiated naive animals. Application of squid homogenate to the oral veil of naive hungry animals (or unpaired controls) evoked synaptic excitation and bursting of PCNs and induced the FMP. In contrast, recordings from paired animals revealed pronounced synaptic inhibition of the PCNs, sufficiently strong to suppress rhythmic bursting activity and block initiation of the FMP. The response of PCNs from untrained satiated animals was indistinguishable from that of the paired animals. Thus, at both behavioral and neural circuit levels, food satiation and aversive chemosensory conditioning appear to have much in common in Pleurobranchaea. Although the motivational and learning effects are not completely identical and later studies were able to dissociate the two to some extent (Kovac, Davis, Matera, Morielli, \& Croll, 1985; Kovac, Matera, Volk, \& Davis, 1986), their similarity should be recognized. And because it is possible that food-shock pairings might lead to greater food ingestion than unpaired training does (see Farley \& Alkon, 1987, for a discussion), the possibility that what may appear to be a primary consequence of associative learning is, instead, a secondary, motivational one should not be dismissed preemptively. 


\section{The Combined Effects of Differential Habituation, Sensitization, and Protection From Habituation Can Be Difficult to Distinguish From Associative Learning}

The lack of stimulus specificity during chemosensory conditioning with molluscs is not inevitable, since considerable specificity has been observed in several reports (Colwill, Absher, \& Roberts, 1988; Farley et al., 1990; Sahley, Gelperin, \& Rudy, 1981; Susswein, Schwarz, \& Feldman, 1986). However, a key question is whether stimulus specificity is necessarily indicative of associative learning. One ambiguity that arises in many reports of reduced feeding in aversive chemosensory conditioning paradigms with molluscs is whether the pairing- and stimulus-specific changes reflect new, associatively acquired hedonic preferences (e.g., aversion to a particular food or odor) or nonassociative amplification of neophobic and/or defensive withdrawal reactions. Most of the molluscan species that have been subjects in chemosensory aversion studies are relatively omnivorous. Hence, rapid associative flavor aversion learning and neophobia might be expected to complement one another in the natural environment and make up an important part of these animals' integrative strategy for coping with the costs entailed by an open feeding system, such as increased likelihood of toxicosis or attack by other animals that are potential meals. A corollary to this is that teasing apart the separate influences of associative learning and neophobia in aversion paradigms may be difficult.

An important consequence of exposure to strong aversive stimulation in many animals is the suppression of feeding directed toward novel food substances: aversive potentiation of neophobia (Braverman \& Jarvis, 1978; Mitchell, Scott, \& Mitchell, 1977; Rzoska, 1953). A second consequence is sensitization, generally regarded as a form of nonassociative learning in which an animal's responses to many external stimuli are strengthened, especially defensive reflexes. When a strong noxious stimulus is presented to molluscs, they often respond with escape or withdrawal from a variety of previously weak or neutral stimuli (Abraham \& Willows, 1971; Balaban, 1983; Pinsker, Henning, Carew, \& Kandel, 1973). Tests of feeding behavior in molluscs, usually accomplished by the manual presentation of pieces of food to an animal's mouth region, elicit competing behaviors of ingestion and withdrawal. If the animal is hungry enough, feeding and ingestion win out. If the animal is satiated or aversively stimulated, withdrawal usually dominates. However, the hunger/satiation state can interact with appetitive and noxious stimuli to determine feeding and avoidance responses. In Pleurobranchaea, where these interactions have been most thoroughly studied, low concentrations of food stimuli may elicit withdrawal and avoidance responses in animals whose feeding thresholds are elevated, because of either satiation or other hormonal influences. Conversely, noxious chemosensory stimuli that normally elicit withdrawal behaviors may elicit feeding behavior in animals with low feeding thresh- olds for food (e.g., because of extreme hunger; Gillette, Huang, Hatcher, \& Moroz, 2000).

Although they are distinguishable, enhanced chemosensory neophobia and sensitized withdrawal from oral tactile stimulation can be expected to combine to suppress ingestion in standard tests of feeding behavior in molluscs. Both can arise from mere exposure to strong aversive stimulation; pairing a chemosensory CS with aversive stimulation is not required. Moreover, if one or more CS is repeatedly presented during sessions in which aversive stimulation is delivered and these CSs themselves evoke learning and/or motivational changes, interactions with the aversive US can occur. The CS-evoked motivational changes, for example, could either add or subtract from the increased arousal/sensitization produced by strong aversive stimulation.

From the aforementioned considerations, aversive chemosensory conditioning paradigms with molluscs might be expected to produce the following. First, pairing a novel chemosensory cue with an aversive stimulus might constitute a functionally more aversive experience for an animal than would explicitly unpaired presentations of the same two stimuli, since novel chemosensory cues often elicit avoidance reactions in sensitized animals and, thus, themselves satisfy the operational definition of an aversive stimulus. Therefore, differences in aversive motivational state could result from paired versus unpaired training conditions and may result in corresponding differences in feeding suppression. Second, unpaired presentations of food and an aversive stimulus potentially allow for both greater habituation to the aversive stimulus and greater habituation of withdrawal reflexes elicited by the chemosensory cue. Thus, the combination of greater aversive motivation and the reduced habituation of withdrawal reactions produced by pairings would be expected to lead to enhanced withdrawal from oral stimulation - that is, reduced feeding. In this way, pairings of a chemosensory cue and an aversive stimulus could produce a partially selective suppression of appetitive behavior directed toward the chemosensory cue, even though no association between that cue and the aversive stimulus had been formed. Is there any evidence that such processes occur in chemosensory aversion conditioning with molluscs? Consider the results from Limax and Aplysia.

In odor aversion learning studies with Limax (Sahley, Gelperin, \& Rudy, 1981; Sahley, Rudy, \& Gelperin, 1981), slugs' odor preferences were tested 2 or $24 \mathrm{~h}$ following aversion conditioning or control stimulation. In most experiments, associatively conditioned animals were exposed to a single conditioning trial in which they were allowed to feed on carrot or potato for $2 \mathrm{~min}$, followed by a 20-min exposure to quinidine sulfate, which elicited copious mucus secretion, attempts at withdrawal, and writhing behavior. Posttraining odor preferences were assessed during six consecutive 60 -sec exposures to a two-odor choice situation. On each of these choice trials, a slug was placed over an elevated and perforated floor in the middle of a circular plastic dish. Underneath one 
side of the floor were filter papers soaked in either carrot or potato food extract solution. Underneath the other side were filters soaked in a familiar "safe" food solution (i.e., rat chow), which the slugs had been previously maintained on in their home cages. A narrow zone containing no odor separated the two sides. The measure of preference was the percentage of time the slug spent over the test (carrot or potato) odor versus the familiar, safe, rat chow odor.

Sahley, Gelperin, and Rudy (1981, Experiment 1) reported that slugs that fed on potato, followed by quinidine exposure (PQ animals), spent only $\sim 12 \%$ of their time over the potato odor. In contrast, control slugs that ingested potato, followed by exposure to saline (PS animals), spent $\sim 80 \%$ of their time over the potato. When tested with carrot versus rat chow odors, however, the PQ and the PS animals spent $\sim 45 \%$ and $\sim 58 \%$ of their time over the carrot, respectively. Thus, only the PQ animals showed a clear avoidance of the potato odor. This aversion was relatively specific, in that the PQ animals were relatively indifferent to carrot.

In their second experiment, Sahley, Gelperin, and Rudy (1981) addressed the issue of pairing specificity for quinidine's effects. Four treatment conditions were compared. Slugs in groups PQ and PS were initially allowed to feed on carrot for $2 \mathrm{~min}$, without experiencing aversive stimulation. Two other groups of slugs (CQ and CS) were allowed to feed on potato for $2 \mathrm{~min}$ and also received no quinidine. Twelve hours after this initial unpunished feeding trial, the PQ slugs were exposed to a single potato-quinidine pairing, whereas the CQ slugs were exposed to a single carrot-quinidine pairing. The PS and the CS slugs were exposed to a potato-saline pairing and a carrot-saline pairing, respectively. The PQ and CQ slugs spent little time over the odor that had been paired with quinidine ( $\sim 6 \%$ to potato and $\sim 13 \%$ to carrot, respectively), whereas the PS and CS animals spent considerable time over these odors $(\sim 68 \%-74 \%$, respectively). In addition, quinidine-stimulated animals (PQ and CQ) spent considerable time over the odor that had not been experienced in conjunction with quinidine $(\sim 68 \%$ to carrot for the PQ and $\sim 64 \%$ to potato for the CQ animals, respectively). In sum, the odor preferences of the PQ and CQ slugs were selectively reduced to the odor that had been paired with quinidine. Was it entirely selective? There are hints that it was not. As in the first experiment, preference for carrot was somewhat smaller for the PQ $(\sim 68 \%)$ than for the PS $(\sim 88 \%)$ animals. And preference for potato was slightly smaller for the CQ $(\sim 66 \%)$ than for the CS ( $74 \%)$ animals. These differences were nonsignificant, although the relatively small $n \mathrm{~s}$ (4) should be kept in mind. A third experiment repeated the procedure of Experiment 1 (PQ and PS training), with the exception that the slugs were exposed only to potato odor and were not allowed to feed on potato. The intent here was to determine whether gustatory (taste) stimulation potentiated the odor aversion (Rusiniak, Hankins, Garcia, \& Brett, 1979). It did not. In summary,
Sahley, Gelperin, and Rudy (1981) demonstrated that pairing a food odor with quinidine resulted in a pairingand stimulus-specific reduction in Limax's preference for that odor. They further concluded that the change in odor preference resulted from associative learning.

In a second series of experiments, Sahley, Rudy, and Gelperin (1981) extended their odor aversion paradigm to second-order conditioning. Slugs exposed to pairings of carrot odor with quinidine (first-order conditioning), followed by pairings of potato with carrot (second-order conditioning), spent significantly less time over both carrot and potato when each was tested against the familiar rat chow odor. In contrast, animals that received the initial carrot-quinidine pairings, followed by unpaired presentations of carrot and potato, displayed a slight preference for potato. Similarly, animals that received initial unpaired presentations of carrot and quinidine, followed by paired presentations of potato and carrot, also exhibited a slight preference for potato. These results clearly indicate that the animals exhibiting an aversion to potato did so because potato had been paired with carrot, which in turn had been paired with aversive quinidine sulfate. The most straightforward interpretation of these results is the one offered by Sahley, Rudy, and Gelperin: The reduced preferences for both carrot and potato were the result of associative learning (first- and second-order conditioning, respectively).

An alternative explanation is that the reduced preferences represented aversive amplification of neophobic reactions to the odors (and/or sensitized withdrawal responses). Perhaps slugs, sensitized by exposures to quinidine, avoid any relatively unfamiliar odor, especially if an alternative safe source of olfactory stimulation is present. Exposure to the novel odor, in the absence of quinidine, would be expected to render the novel odor $f a$ miliar (habituation of neophobia). Thus, slugs exposed to pairings of carrot with quinidine may fail to habituate in their neophobic reactions to carrot. Indeed, there may be genuine amplification of the neophobia, due to sensitization. When tested in the choice procedure pitting carrot against a familiar safe odor (i.e., rat chow), the differential neophobic reactions may lead to reduced preference for the less familiar carrot odor.

This analysis can also be extended to the apparent second-order conditioning of odor aversions observed by Sahley, Rudy, and Gelperin (1981). To the extent that avoidance of the first-order conditioned odor (e.g., carrot) reflects its aversive, sensitizing properties - regardless of how these were acquired - one might expect it to also serve a sensitizing role in the second-order conditioning phase of training, similar to that postulated for quinidine during first-order conditioning. Thus, for animals with previous aversive experience with carrot-quinidine pairings, subsequent repeated exposures to potato-carrot pairings might be expected to produce an amplified neophobic reduction in preference for potato, particularly when tested against the familiar and safe rat chow odor. Control condition animals that received initial carrot- 
quinidine pairings, followed by unpaired carrot and potato odor presentations, might be expected to exhibit an aversion to carrot, but not to potato, because of habituation of neophobia to the latter cue during the second-order conditioning phase of the experiment.

This same kind of analysis can also be extended to contextual conditioning paradigms. Consider studies by Colwill and colleagues (Colwill et al., 1988; Colwill, Goodrum, \& Martin, 1997), who have presented strong evidence for context discrimination learning by Aplysia. In an aversive context-conditioning study using two distinct environments that differed in substrate texture, shape, chemosensory composition, and other cues, Colwill et al. (1988) administered a series of electric shocks in one context $(\mathrm{CS}+)$, but not in the other $(\mathrm{CS}-)$. They found that the reinforced context (1) enhanced the duration of an unconditioned defensive reflex (siphon withdrawal elicited by tactile stimulation, SWR) and (2) reduced the conditioning-produced increases in this reflex when the tactile stimulus was later paired with shock (i.e., a contextual blocking effect). A second experiment showed that the context-shock association could be extinguished (habituated?) by nonreinforced exposure to the formerly reinforced context.

Comparison of median SWR durations for Aplysia tested in the presence of the reinforced $(\mathrm{CS}+)$ versus nonreinforced $(\mathrm{CS}-$ ) contexts indicated significantly longer durations for the former cue: $\sim 70 \mathrm{sec}$ for the CS+ versus $\sim 52 \mathrm{sec}$ for the $\mathrm{CS}-$. But because the preconditioning SWR durations were not reported in their first experiment, the extent to which the CS+ versus CSdifference reflected (1) increases in duration to the CS+, relative to baseline preconditioning levels, presumably reflecting excitatory conditioning to the CS+ or (2) decreases in duration to the $\mathrm{CS}-$, presumably reflecting response-decremental processes elicited by the $\mathrm{CS}-$, is uncertain. Evidence bearing on this issue can be gleaned from the results of their second experiment that demonstrated contextual blocking. Here, preconditioning SWR durations were reported that were elicited by mild tactile siphon stimulation administered in a third context, the home tank. From Figure 2 in Colwill et al. (1988), these durations were $\sim 80-90 \mathrm{sec}$. If these were representative of the preconditioning values obtained in the two distinctive contexts in Experiment 1, it would appear that contextual conditioning decreased the SWR, more so for the unpaired $(\mathrm{CS}-)$ than for the paired $(\mathrm{CS}+)$ context.

The latter issue aside, what is indisputable about Colwill et al.'s (1988) results is that SWR behavior was controlled by the animals' previous experience with the environmental context prevailing at the time of training and testing. Colwill et al. (1988) interpreted this control as indicating that Aplysia had learned specific context-US associations. They pointed out that their results (as well as those of many analogous blocking experiments with vertebrates) did not allow a definitive conclusion to be drawn as to whether the differential control by contextual stimuli was due to excitatory conditioning to the CS + or in- hibitory conditioning to the CS-. But because the two contexts differed in their effects on SWR, they concluded that discriminative conditioning (i.e., associative learning) of some sort had occurred.

Although this interpretation of their results is very plausible, it is not the only possible one. We next will sketch an alternative account of the differential control exerted by context that is similar to the one we suggested might explain the pairing- and stimulus-specific odor preferences observed in discrete-trial aversion-learning experiments in Pleurobranchaea and Limax. This nonassociative account does not assume that associative learning occurred to either the CS + or the $\mathrm{CS}-$.

Assume that exposure of Aplysia to a novel environment, even in the absence of any explicit aversive stimulation, constitutes an arousing/sensitizing event that may enhance the animal's general responsiveness to a variety of external stimuli, including siphon withdrawal elicited by weak tactile stimuli. It is well established that novel environments can arouse vertebrates (Berlyne, 1960). More to the point for molluscs, Kemenes and Benjamin (1994) found that transfer of hungry Lymnaea from their home tank to a novel aquatic environment strongly facilitated spontaneous (as well as sucroseevoked) rasping and ingestional behaviors. They suggested that a novel environment elicits a "curiosity" drive in Lymnaea, similar to that evoked in vertebrates under analogous circumstances (Berlyne, 1960; Konorski, 1967).

For the safe environment (CS-; no shocks) in Colwill et al. (1988), assume further that Aplysia habituated to this context, both within and across their successive daily exposures to this environment, and thus were less aroused at the end of each training session. Conversely, for the context (CS+) in which daily shocks were given, habituation may have been retarded/prevented by the aversive stimulation. The animals may even have been sensitized in this context. It is worth noting that sensitization and arousal in invertebrates - gastropods in particular (Abraham \& Willows, 1971; Wells \& Wells, 1971) —can be conspicuously long-lived, often persisting for days or even weeks (Pinsker et al., 1973).

Thus, postconditioning tests of the SWR in the CS+ and CS- environments can be reconceptualized as tests of a withdrawal reflex in contexts with different capacities to arouse the animal, owing to differences in habituation and/or sensitization. The expected result would be greater responsiveness when the animal is tested in the $\mathrm{CS}+$ context. To be sure, context specificity occurs. But in this account, specificity derives from differences in nonassociative learning.

This nonassociative account of differential control by context is not without precedent. Consider the following characterization of the relationship between arousal, sensitization, habituation, and context (Kandel, 1976): "Animals habituate readily in a safe environment, where they learn quickly to ignore a novel but nonharmful stimulus that is repeatedly presented. An animal that has received 
a few harmful stimuli becomes sensitized; it learns that, for the moment, its environment is no longer completely safe and it will not habituate as readily as an unperturbed animal. But an animal that has received repeated harmful stimuli learns that its environment is dangerous. In the presence of danger, any tendency for habituation of withdrawal reflexes is maladaptive" (pp. 656-657).

The associative versus nonassociative accounts of contextual specificity for Colwill et al.'s (1988) results might be distinguished by comparing the posttraining SWR responses in the presence of a CS + and a CSwith their pretraining baseline levels. If, for example, the posttraining responses in both contexts were weaker than that at the baseline level, this would seem to support the nonassociative account. Although habituation of arousal would evidently have occurred to both contexts, selective attenuation of habituation by shock in the reinforced context would have led to less of a response decrement when the SWR response was tested in the CS + context. Alternatively, if posttraining responses to a $\mathrm{CS}+$ and a CS - both proved greater than that at the baseline level, the interpretation would be more uncertain. On the one hand, such a result would be the one expected under the assumption that associative excitatory contextual conditioning had occurred to the $\mathrm{CS}+$, perhaps with some stimulus generalization to the $\mathrm{CS}-$. However, the same outcome might be expected if the electric shocks had produced a persistent sensitization/facilitation of arousal to the $\mathrm{CS}+$ environment (again with some generalization to the CS-). Unfortunately, there seems to be no pattern of results that would uniquely support the associativelearning interpretation, although some patterns could rule it out. An additional complexity is that numerous interpretations are possible if one also allows for inhibitory conditioning to occur to a CS- .

In view of this indeterminacy, it may be more practical to look for convergent outcomes from other tests in seeking a resolution of this issue. For example, if no difference in baseline SWR responses could be detected for Aplysia tested in the presence of equally distinctive novel versus familiar contexts, there would be little grounds for invoking a habituation of arousal process in animals repeatedly exposed to the (nonshocked) CS - context. Similarly, if shocking animals just prior to their placement in a novel context failed to increase the strength of their SWR reflex above the level occurring for nonshocked animals, invoking sensitization of context-evoked arousal by shock would be specious. Even if direct evidence for these nonassociative processes should be found, additional features of the results (time course, persistence, generalization to other contexts, etc.) might constrain their applicability to the outcomes of differential conditioning studies. Colwill (1996) has outlined additional incisive strategies for addressing these and related issues that should also prove informative.

Before addressing Colwill et al.'s (1988) very interesting report of context blocking in Aplysia, two limitations on what has been demonstrated so far should be recognized. First, as Colwill et al. (1988) noted, the impaired learning to a tactile CS that was observed when conditioning was conducted in a previously reinforced context was "statistically rather modest" (p. 4436). Second, blocking effects with discrete-trial, punctuate CS presentations have not yet been reported for Aplysia. The context-blocking study of Colwill et al. (1988) is the sole report of blocking effects with this species. Thus, it may be premature to conclude that the impaired performance to the contextually blocked CS in this study reflects the same processes occurring in discrete-trial blocking studies with vertebrates.

How might Colwill et al.'s (1988) context-blocking results be integrated within the preceding nonassociative account? One way would be to acknowledge that although the previously reinforced environment interfered with excitatory conditioning to the tactile CS, this interference derived from the intrinsic aversive/arousing characteristics of the context, rather than from any acquired via associative learning (i.e., the signaling of electric shock). Thus, learning that a tactile CS is associated with electric shock may be disrupted if the conditioning is carried out in an environment that is highly aversive and/or arousing, regardless of how it came to be that way. In this view, the unconditioned reactions to the contextual CS + and their modification by nonassociative learning (e.g., facilitated/sensitized arousal) would be viewed as responsible for blocking. Maybe an aversive/ arousing electric shock US is processed differently and/or less effectively and is, therefore, less able to support conditioning if it is presented within an aversive/arousing context (e.g., a negative "priming" effect, Wagner, 1976; a nonoptimal level of arousal, Yerkes \& Dodson, 1908).

What processes could conceivably underlie such effects? Perhaps the contextual CS + acts as a kind of tonic, background, adapting stimulus, diminishing activation of neural circuits activated during aversive Pavlovian conditioning that respond to polymodal, arousing, threatening stimuli. Alternatively, high arousal might diminish animals' sensitivity to the tactile CS or distract attention from it.

\section{Nonassociative Influences During Appetitive Chemosensory Conditioning in Molluses}

Appetitive chemosensory learning paradigms in molluscs have sometimes been suggested as less likely to entail the strong nonassociative sensitization-like processes that aversive paradigms undoubtedly do. This is because in molluscs, as in most other animals, escape reactions are at the top of the behavioral hierarchy (Davis et al., 1974; Everett, Ostfield, \& Davis, 1982) and are, thus, easily expressed and maintained. Consistent with this idea is the observation that food avoidance learning in gastropods is generally more readily achieved than appetitive conditioning (Mpitsos \& Davis, 1973; Sahley, Martin, \& Gelperin, 1990, 1992; but cf. Whelan \& McCrohan, 1996). However, these results should not be interpreted as indicating that appetitive paradigms are entirely free of non- 
associative processes. In our view, the relative importance of nonassociative versus associative learning processes in appetitive-conditioning paradigms needs to be established on a case-by-case basis.

Using a Pavlovian appetitive discrimination-learning paradigm with Aplysia, Colwill et al. (1997) obtained evidence for selective responding to discrete $\mathrm{CS}+\mathrm{s}$. These experiments are noteworthy for two reasons. First, greater responding to a $\mathrm{CS}+$ than to a $\mathrm{CS}$ - was consistently observed, regardless of the stimuli assigned as the $\mathrm{CS}+$ and the $\mathrm{CS}-$. Second, discrimination reversal was observed when tactile cues (rough vs. smooth syringe tips applied to the lips) were used as the $\mathrm{CS}+$ and the $\mathrm{CS}-$. Animals exposed to a chemosensory stimulus (e.g., lemon-laced ASW) paired with food showed conditioned biting responses to that $\mathrm{CS}+$, but much less responding to a second stimulus (e.g., a banana) that was never paired with edible food $(\mathrm{CS}-)$. Fourteen full discrimination-training sessions, with six trials each for $\mathrm{CS}+$ and $\mathrm{CS}-$ per session, were given. Testing was conducted $1 \mathrm{~h}$ after a 15 th half-session and involved two nonreinforced presentations each of $\mathrm{CS}+$ and $\mathrm{CS}-$. During the final half-session, the mean percentage of $\mathrm{CS}+$ and $\mathrm{CS}-$ trials with a bite response was $94 \%$ and $73 \%$, respectively. During the nonreinforced tests, the comparable values were $\sim 89 \%$ and $\sim 64 \%$. Although these differences in responding to the $\mathrm{CS}+$ versus the $\mathrm{CS}-$ were statistically significant, it is worth noting that responses to the CS- were $\sim 70 \%$ of the levels to the $\mathrm{CS}+$. In addition, prior to conditioning, the tactile/chemosensory cues elicited biting on $\sim 20 \%$ of the trials.

As was true for aversive chemosensory conditioning in Aplysia (Colwill et al., 1988), a degree of pairing and stimulus specificity was obtained for appetitive chemosensory learning. However, the level of maintained responding to the CS - was considerable and clearly elevated above baseline. The relatively weak stimulus specificity in this experiment could have been due to stimulus generalization, since the $\mathrm{CS}+$ and the $\mathrm{CS}$ - shared common tactile components, involving stimulation of lips, mouth, and oral veil and, perhaps, common chemosensory elements as well.

A nonassociative, differential habituation/sensitization account of these appetitive-conditioning results can be formulated, similar to the one formulated for aversive conditioning. First, note that prior to conditioning, the CSs used (flavored seawater) elicit unconditioned responses (URs) that are similar to those elicited by the US of food. The strength and probability of occurrence of these responses for an animal would be expected to vary with the animal's level of hunger, general arousal, and so forth. These fractional URs (biting, rasping movements of the mouth, etc.) probably derive from the common stimulus elements that the CSs and the USs share: tactile stimulation of lips, mouth, and oral veil regions and common chemical constituents. These responses habituate with repeated testing, if no strong, motivationally significant US is presented. Food, the US used, induces an arousal state in many gastropods (Kemenes \& S.-Rózsa,
1987; Lee \& Palovcik, 1976; Tuersley \& McCrohan, 1987)_including Aplysia (Advokat, 1980; Susswein, Weiss, \& Kupfermann, 1978; Weiss, Koch, Koester, Rosen, \& Kupfermann, 1982) - that facilitates various appetitive behaviors, such as bite/strike and rasping movements of the mouth. In addition to facilitating the biting responses to both the $\mathrm{CS}+$ and the $\mathrm{CS}-$, the US also plausibly disrupts habituation to the CS+ with which it is paired. Thus, the US of food serves to increase and maintain the appetitive (unconditioned) behaviors elicited by both the $\mathrm{CS}+$ and the $\mathrm{CS}-$. And pairings of $\mathrm{CS}+$ and food confer a modicum of selectivity upon the CS+.

\section{Summary}

The studies reviewed here, and our own results with Hermissenda, underscore the fact that the food extracts used as CSs in many chemosensory conditioning experiments with molluscs cannot accurately be regarded as insignificant "neutral" stimuli, as is often the case for the stimuli chosen as CSs in conditioning studies with vertebrates. They are, in fact, USs. Prior to any conditioning, these chemosensory stimuli elicit overt and conspicuous behaviors, such as feeding and disruption of escape locomotion (e.g., Figure 4C in Walters, Carew, \& Kandel, 1979). Food (solid or extract) has been used as an US to condition feeding responses to other CSs - typically, novel chemical or tactile stimuli (Audesirk et al., 1982; Colwill et al., 1997; Kemenes \& Benjamin, 1989; Mpitsos \& Davis, 1973) — and to countercondition a preference for an innately aversive odor in Limax (Sahley et al., 1990). In short, food extracts are powerful stimuli that affect multiple sensory, motivational, and behavioral systems in gastropod molluscs. Whether used as a CS or a US in chemosensory conditioning paradigms, food can have manifold nonassociative effects and can alter behavioral hierarchies in ways that complicate the analysis of associative learning.

The use of aversive USs in many chemosensory conditioning paradigms with molluscs entails its own additional problems. Strong electric shock can suppress feeding, probably through a variety of nociceptive and nonnociceptive processes. Two deserve special attention here. Considerable evidence suggests that the central motivational states of "hunger" and "pain" are reciprocally inhibitory (Dickinson \& Pearce, 1977; Gillette et al., 2000). Stimuli that trigger active escape behavior in molluscs generally inhibit feeding (Davis et al., 1974), whereas stimuli that initiate feeding in hungry animals can interfere with escape behavior (Ram et al., 1988; Walters et al., 1979). Although these motivational interactions are not unique to molluscs, having been observed in many vertebrate species as well (Dickinson \& Pearce, 1977) and having figured prominently in theories of learning that emphasize the conditioning of central motivational states (Rescorla \& Solomon, 1967), they are particularly clear and strong in the gastropods.

Disentangling the relative contributions of associative and nonassociative processes to the outcomes of condi- 
tioning experiments with molluscs, especially those involving chemosensory stimuli, can be deceptively complex. This perspective not only applies to the results of simple single-CS procedures but also extends to the analysis of many multiple-CS phenomena, including several typically considered to be "hallmarks" of associative learning, such as discriminative conditioning, blocking, and so forth. These nonassociative processes can arise from the stimuli used as CSs, USs, or both.

\section{REFERENCES}

Abraham, F. D., \& Willows, A. O. D. (1971). Plasticity of a fixed action pattern in the sea slug Tritonia diomedea. Communications in Behavioral Biology, 6, 271-280.

Advokat, C. (1980). Modulation of defensive reflexes in Aplysia californica by appetitive stimulation. Behavioral \& Neural Biology, $\mathbf{2 8}_{2}$ 253-265.

ALKON, D. L. (1974). Associative training of Hermissenda. Journal of General Physiology, 64, 70-84.

ALKON, D. L., AKAIKE, T., \& HARRIGAN, J. (1978). Interaction of chemosensory, visual, and statocyst pathways in Hermissenda crassicornis. Journal of General Physiology, 71, 177-194.

AUdesirk, T., AleXander, J. E., JR., AUdesirk, G., \& Moyer, C. M. (1982). Rapid, nonaversive conditioning in a freshwater gastropod: I. Effects of age and motivation. Behavioral Neural Biology, 36, 379390.

Balaban, P. M. (1983). Postsynaptic mechanisms of withdrawal reflex sensitization in the snail. Neurobiology, 14, 365-375.

Berlyne, D. E. (1960). Conflict, arousal, and curiosity. New York: McGraw-Hill.

Bitterman, M. E., Menzel, R., Fietz, A., \& Schafer, S. (1983). Classical conditioning of proboscis extension in honeybees (Apis mellifera). Journal of Comparative Psychology, 97, 107-119.

BraVERMAN, N. S., \& JARVIS, P. S. (1978). Independence of neophobia and taste aversion learning. Animal Learning \& Behavior, 6, 406-412.

ColwiLl, R. M. (1996). Detecting associations in Pavlovian conditioning and instrumental learning in vertebrates and invertebrates. In C. F. Moss \& S. J. Shettleworth (Eds.), Neuroethological studies of cognitive and perceptual processes (pp. 31-62). Boulder, CO: Westview.

Colwill, R. M., Absher, R. A., \& Roberts, M. L. (1988). Context-US learning in Aplysia californica. Journal of Neuroscience, 8, 4434-4439.

Colwill, R. M., Goodrum, K., \& MarTin, A. (1997). Pavlovian appetitive discriminative conditioning in Aplysia californica. Animal Learning \& Behavior, 25, 268-276.

Davis, W. J., MPITsos, G. J., \& Pinneo, J. M. (1974). The behavioral hierarchy of the mollusk Pleurobranchea. Journal of Comparative Physiology, 90, 207-224.

Davis, W. J., Villet, J., Lee, D., Rigler, M., Gillette, R., \& Prince, E. (1980). Selective and differential avoidance learning in the feeding and withdrawal behaviors of Pleurobranchea. Journal of Comparative Physiology, 138A, 157-165.

DiCKINSON, A., \& PEARCE, J. M. (1977). Inhibitory interactions between appetitive and aversive stimuli. Psychological Bulletin, 84, 690-711.

Everett, R. A., OstField, R. S., \& Davis, W. J. (1982). The behavioral hierarchy of the garden snail Helix aspersa. Zeitschrift für Tierpsychologie, 59, 109-126.

FARLEY, J. (1987). [The use of electric shock as a US in Pavlovian conditioning of chemosensory aversions and phototactic suppression in Hermissenda]. Unpublished raw data.

FARLEY, J., \& AlKoN, D. L. (1987). Cellular analysis of gastropod learning. In A. J. Greenberg (Ed.), Cell receptors and cell communication (pp. 220-266). Basel: Karger.

Farley, J., Grover, L. M., Sun, L., Huang, S. S., Eisthen, H. L., Girolami, C., \& Wu, R. (1990). Chemosensory conditioning of Hermissenda crassicornis. Behavioral Neuroscience, 104, 583-596.

FARLEY, J., \& JiN, I. (1997). Potentiation of phototactic suppression in
Hermissenda by compound conditioning results in potentiated excitability changes in type B and A photoreceptors. Behavioral Neuroscience, 111, 309-319.

FaRLEY, J., R EASONER, H., \& JANSSEn, M. (1997). Potentiation of phototactic suppression in Hermissenda by a chemosensory stimulus during compound conditioning. Behavioral Neuroscience, 111, 320341.

GELPERIN, A. (1975). Rapid food-aversion learning by a terrestrial mollusk. Science, 189, 567-570.

Gillette, R., Huang, R. C., Hatcher, N., \& Moroz, L. L. (2000). Cost-benefit analysis potential in feeding behavior of a predatory snail by integration of hunger, taste, and pain. Proceedings of the $\mathrm{Na}$ tional Academy of Sciences, 97, 3585-3590.

Gillette, R., Kovac, M. P., \& Davis, W. J. (1978). Command neurons in Pleurobranchaea receive synaptic feedback from the motor network they excite. Science, 199, 798-801.

Grover, L., FARLeY, J., \& VOLD, L. (1987).Training and testing determinants of short-term associative suppression of phototaxic behavior in Hermissenda. Behavioral \& Neural Biology, 47, 275-306.

Hanson, H. M. (1959). Effects of discrimination training on stimulus generalization. Journal of Experimental Psychology, 58, 321-334.

Hildebrand, J. G., \& SHEPHERd, G. M. (1997). Mechanisms of olfactory discrimination: Converging evidence for common principles across phyla. Annual Review of Neuroscience, 20, 595-631.

JENKINS, H. M., \& HaRRISON, R. H. (1960). Effects of discrimination training on auditory generalization. Journal of Experimental Psychology, 59, 246-253.

JENKINS, H. M., \& HARRISON, R. H. (1962). Generalization gradients of inhibition following auditory discrimination training. Journal of the Experimental Analysis of Behavior, 5, 435-441.

JiN, I., HuANG, H., KIM, J.-I., \& FARLEY, J. (2004). Chemosensory conditioning in molluscs: I. Failure of chemosensory conditioning in Hermissenda. Learning \& Behavior, 32, 257-276.

KANDEL, E. R. (1976). Cellular basis of behavior. San Francisco: Freeman. Kemenes, G., \& Benjamin, P. R. (1989). Appetitive learning in snails shows characteristics of conditioning in vertebrates. Brain Research, 489, 163-166.

Kemenes, G., \& Benjamin, P. R. (1994). Training in a novel environment improves the appetitive learning performance of the snail, Lymnaea stagnalis. Behavioral \& Neural Biology, 61, 139-149.

Kemenes, G., \& S.-Rózsa, K. (1987). The role of serotonergic mechanisms in food-induced arousal of the snail Helix pomatia L. In H. H. Boer, W. P. M. Geraerts, \& J. Joosse (Eds.), Neurobiology: Molluscan models (pp. 277-287). Amsterdam: North-Holland.

KONORSKI, J. (1967). Integrative activity of the brain. Chicago: University of Chicago Press.

Kovac, M. P., Davis, W. J., Matera, E. M., Morielli, A., \& Croll, R. P. (1985). Learning: Neural analysis in the isolated brain of a previously trained mollusc, Pleurobranchaea californica. Brain Research, 331 $\underline{275-284}$.

Kovac, M. P., Matera, E. M., Volk, P. J., \& Davis, W. J. (1986). Food avoidance learning is accompanied by synaptic attenuation in identified interneurons controlling feeding behavior in Pleurobranchaea. Journal of Neurophysiology, 56, 891-905.

Kristal, M. B., Steuer, M. A., Nishita, J. K., \& Peters, L. C. (1980). Neophobia and water intake after repeated pairings of novel flavors with toxicosis. Physiology \& Behavior, 24, 979-982.

Kupfermann, I., \& Pinsker, H. (1968). A behavioral modification of the feeding reflex in Aplysia californica. Communications in Behavioral Biology, 2, 13-17.

Lee, R. M., \& PalovciK, R. A. (1976). Behavioral states and feeding in the gastropod Pleurobranchaea. Behavioral Biology, 16, 251-266.

Mitchell, D., Scott, D. W., \& Mitchell, L. K. (1977). Attenuated and enhanced neophobia in the taste-aversion "delay of reinforcement" effect. Animal Learning \& Behavior, 5, 99-102.

Mook, D. G. (1990). Satiety, specifications, and stop rules: Feeding as voluntary action. In A. N. Epstein \& A. R. Morrison (Eds.), Progress in psychobiology and physiological psychology 14 (pp. 1-65). New York: Academic Press.

Moore, J. W. (1972). Stimulus control: Studies of auditory generaliza- 
tion in rabbits. In A. H. Black \& W. F. Prokasy (Eds.), Classical conditioning II: Current research and theory (pp. 206-230). New York: Appleton-Century-Crofts.

Mpitsos, G. J., Collins, S. D., \& McClellan, A. D. (1978). Learning: A model system for physiological studies. Science, 199, 497-506.

Mpitsos, G. J., \& Davis, W. J. (1973). Learning: Classical and avoidance conditioning in the mollusk Pleurobranchaea. Science, $\mathbf{1 8 0}$, 317-320.

Mustaca, A. E., Gabelli, F., Papini, M. R., \& Balsam, P. (1991). The effects of varying the interreinforcement interval on appetitive contextual conditioning. Animal Learning \& Behavior, 19, 125-138.

Nelson, M. C. (1971). Classical conditioning in the blowfly (Phormia regina). Journal of Comparative \& Physiological Psychology, $\mathbf{7 7}_{2}$ 353-368.

Pinsker, H., Henning, W. A., Carew, T. J., \& Kandel, E. R. (1973). Long-term sensitization of a defensive withdrawal reflex in Aplysia. Science, 182, 1039-1042.

RaM, J. L., NoIrot, G., Waddell, S., \& Anderson, M. (1988). Singleness of action in the interactions of feeding with other behaviors in Hermissenda crassicornis. Behavioral \& Neural Biology, 49, 97111.

ResCoRla, R. A., \& Solomon, R. L. (1967). Two-process learning theory: Relations between Pavlovian conditioning and instrumental learning. Psychological Review, 74, 151-182.

RiLLING, M. R. (1977). Stimulus control and inhibitory processes. In W. K. Honig \& J. E. R. Staddon (Eds.), Handbook of operant behavior (pp. 432-480). Englewood Cliffs, NJ: Prentice-Hall.

Rogers, R. F., \& MAtzel, L. D. (1995). Higher-order associative processing in Hermissenda suggests multiple sites of neuronal modulation. Learning \& Memory, 2, 279-298.

Rogers, R. F., SCHILler, K. M., \& MATZEL, L. D. (1996). Chemosensorybased contextual conditioning in Hermissenda crassicornis. Animal Learning \& Behavior, 24, 28-37.

Rusiniak, K. W., HankinS, W. G., Garcia, J. G., \& Brett, L. P. (1979). Flavor-illness aversions: Potentiation of odor by taste in rats. Behavioral \& Neural Biology, 25, 1-7.

RzosKa, J. (1953). Bait shyness, a study in rat behaviour. British Journal of Animal Behaviour, 1, 128-135.

Sahley, C., Gelperin, A., \& Rudy, J. (1981). One-trial associative learning modifies food odor preferences of a terrestrial mollusk. Proceedings of the National Academy of Sciences, 78, 640-642.

Sahley, C., Martin, K. A., \& Gelperin, A. (1990). Analysis of associative learning in the terrestrial mollusk Limax maximus: II. Appetitive learning. Journal of Comparative Physiology A, 167, 339-345.

Sahley, C., Martin, K. A., \& GelPerin, A. (1992). Odors can induce feeding motor responses in the terrestrial mollusk Limax maximus. Behavioral Neuroscience, 106, 563-568.

SaHLEY, C., Rudy, J., \& GeLPERIN, A. (1981). An analysis of associa- tive learning in a terrestrial mollusc: I. Higher-order conditioning, blocking and a transient US pre-exposure effect. Journal of Comparative Physiology $A, \mathbf{1 4 4}, 1-8$.

SuSSWEIN, A. J., SchWARZ, M., \& FELdMAN, E. (1986). Learned changes of feeding behavior in Aplysia in response to edible and inedible foods. Journal of Neuroscience, $\mathbf{6}, 1513-1527$.

Susswein, A. J., WeIss, K. R., \& KUPFERMANN, I. (1978). The effects of food arousal on the latency of biting in Aplysia. Journal of Comparative Physiology, 123, 31-41.

Sutherland, N. S., \& Mackintosh, N. J. (1971). Mechanisms of animal discrimination learning. New York: Academic Press.

TalK, A. C., Muzzio, I. A., \& Matzel, L. D. (1999). Neurophysiological substrates of context conditioning in Hermissenda suggest a temporally invariant form of activity-dependent neuronal facilitation. Neurobiology of Learning \& Memory, 72, 95-117.

Thомаs, D. R. (1970). Stimulus selection, attention, and related matters. In J. H. Reynierse (Ed.), Current issues in animal learning (pp. 311-356). Lincoln: University of Nebraska Press.

Tuersley, M. D., \& McCrohan, C. R. (1987). Food arousal in the pond snail, Lymnaea stagnalis. Behavioral \& Neural Biology, 48, 222-236.

WAGNER, A. R. (1976). Priming in STM: An information processing mechanism for self-generated or retrieval-generated depression in performance. In T. J. Tighe \& R. N. Leaton (Eds.), Habituation: Perspectives from child development and animal behavior (pp. 95-128). Hillsdale, NJ: Erlbaum.

Walters, E. T., Carew, T. J., \& Kandel, E. R. (1979). Classical conditioning in Aplysia. Proceedings of the National Academy of Sciences, 76, 6675-6679.

Weiss, K. R., Koch, U. T., Koester, J., Rosen, S. C., \& Kupfermann, I. (1982). The role of arousal in modulating feeding behavior of Aplysia: Neural and behavioral studies. In B. G. Hoebel \& D. Novin (Eds.), The neural basis of feeding and reward (pp. 25-27). Brunswick, ME: Haer Institute.

Wells, M. J., \& Wells, J. (1971). Conditioning and sensitization in snails. Animal Behavior, 19, 305-312.

Whelan, H. A., \& McCrohan, C. R. (1996). Food-related conditioning and neuronal correlates in the freshwater snail Lymnaea stagnalis. Journal of Molluscan Studies, 62, 483-494.

Williams, D. A., Frame, K. A., \& LoLordo, V. M. (1991). Reexamination of contextual conditioning with massed versus distributed unconditioned stimuli. Journal of Experimental Psychology: Animal Behavior Processes, 17, 202-209.

YeRKES, R. M., \& DoDSON, J. D. (1908). The relation of strength of stimulus to rapidity of habit-formation. Journal of Comparative Neurological Psychology, 18, 459-482.

(Manuscript received September 22, 2003; revision accepted for publication April 17, 2004.) 